\title{
REBA
}

Revista Boliviana de Administración

Volumen 1 | Número 1 | Enero-junio 2019 | ISSN: 2710-0863

https://doi.org/10.33996/reba.vlil.2

\section{Análisis del liderazgo organizacional en el sector hotelero. Caso de estudio Hotel Las Cabañas, municipio Los Guayos, estado Carabobo}

\section{Organizational leadership analysis in the hotel sector. Case study Hotel Las Cabañas, Municipality Los Guayos, Carabobo State}

\author{
$\checkmark$ Baptista Carmelina \\ carmelo_baptista@hotmail.com \\ ORCID: 0000-0003-2257-944X \\ Universidad de Carabobo. Venezuela
}

\author{
4 Pérez Mayibel \\ mayibel_16@hotmail.com \\ ORCID: 0000-0002-9734-5624 \\ Universidad de Carabobo. Venezuela
}

\author{
$\triangle$ Prince Gilberto \\ geprincea@hotmail.com \\ ORCID: 0000-0001-8106-5639 \\ Universidad de Carabobo. Venezuela
}

Artículo recibido en julio 2018 / Arbitrado en agosto 2018 / Publicado en enero 2019

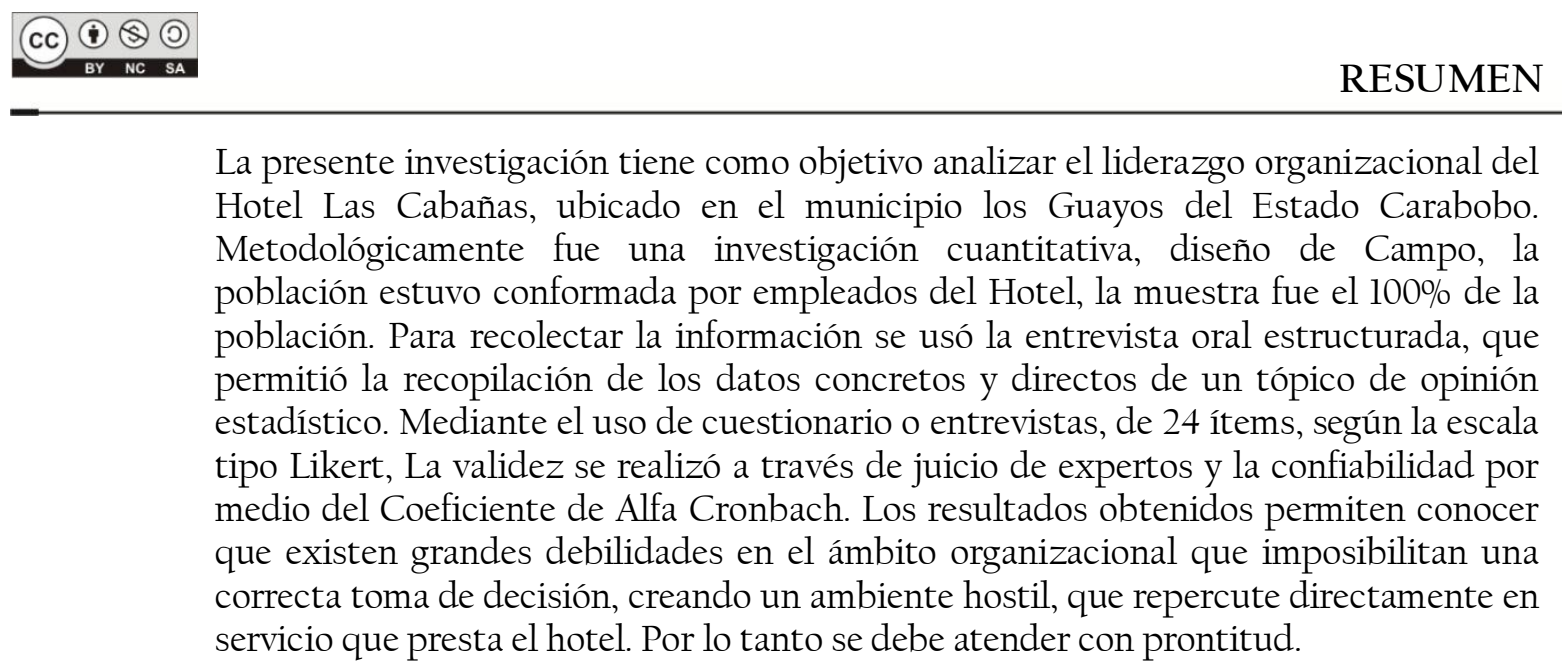

Palabras clave: Análisis, Hotel, Liderazgo Organizacional

ABSTRACT

The objective of this investigation is to analyze the organizational leadership of the Hotel Las Cabañas, located in the Los Guayos municipality of Carabobo State. Methodologically it was a quantitative research, Field design, the population was made up of Hotel employees, the sample was $100 \%$ of the population. To collect the information, a structured oral interview was used, which allowed the collection of concrete and direct data on a topic of statistical opinion. Through the use of a 24-item questionnaire or interviews, according to the Likert-type scale, validity was performed through expert judgment and reliability through the Alpha Cronbach Coefficient. The results obtained allow us to know that there are great weaknesses in the organizational sphere that make correct decision-making impossible, creating a hostile environment that directly affects the service provided by the hotel. Therefore it must be attended to promptly.

Keywords: Analysis, Hotel, Organizational Leadership Organizacional 


\section{INTRODUCCIÓN}

Las organizaciones son unidades sociales dentro de las cuales se establecen relaciones entre los distintos elementos que la integran, a su vez, estas deben contar con una serie de recursos, los cuales administrados eficazmente conducirán al logro de los objetivos trazados; viven constantes cambios que les permite mantener un nivel de competitividad mayor en referencia al desempeño y la calidad del servicio que presten, Sobre este tema Zamora, A. y Poriet, Y. (2012), expresan que:

Las organizaciones han visto la necesidad de redimensionarse, incorporando nuevas estructuras más flexibles, de manera de adaptarse mejor a los entornos internacionales. Esta nueva forma de manejar las organizaciones, responde a la urgente necesidad de focalizar, definir y satisfacer los requerimientos de los clientes. La orientación que predican las organizaciones del siglo XXI, de forma tal de no ser vulnerables, está basada en buscar la aceptación del público; para lo cual han desarrollado programas que las ayuden a ser vistas como verdaderamente responsables, en el cuidado del medio ambiente, y con altos niveles de valores éticos, en la forma de hacer negocios. (p.68)

En tal sentido, una organización se encuentra representada por un grupo de personas con responsabilidades específicas, que actúan en conjunto para lograr alcanzar un propósito determinado que va de la mano con los líderes y el liderazgo que desarrollen. Sobre este tema Rodríguez (2013), expresa:

El liderazgo, a nivel mundial constituye uno de los roles más importantes dentro de la estructura grupal; para alcanzar un buen liderazgo el líder debe disponer de tiempo y hacer contacto directo con las personas de la empresa, con el fin de lograr resultados positivos. (p.32)

Es así como, dentro de toda organización el desempeño del líder juega un papel fundamental, es él quien guía y motiva al personal para que logre cada uno de los objetivos planteados, a través de las labores asignadas. El éxito de una organización empresarial depende del esfuerzo de cada uno de los miembros, motivados a través del liderazgo ejercido en cada trabajador, por ello, este mencionado liderazgo es fundamental, igualmente acota Rodríguez (2013)

El liderazgo representa una de las funciones más exigentes que pueda ser ejercida dentro de cualquier organización, pública o privada, nacional o internacional; por no existir ciertas características únicas que definan la personalidad de los líderes para influir positivamente sobre los seguidores y lograr en ellos eficiencia en la realización de las actividades. (p.32)

Es decir, que los lideres, poseen personalidades diferentes, como seres humanos, tienen patrones de actitudes, pensamientos, sentimientos y conductas diferentes; lo que los lleva a la persistencia y estabilidad a lo largo de la vida de modos diferentes. Por ello, pueden diferir al tomar decisiones o asumir responsabilidades ante una determinada circunstancia; pero convergen en poseer habilidades gerenciales o directivas para influir en la forma de ser o actuar de las personas o en ungrupo de trabajo determinado, haciendo que este equipo trabaje con entusiasmo hacia el logro de las metas y objetivos.

Es de hacer notar, que existen culturas organizacionales diferentes, cada una con 
técnicas y estilos de liderazgo distintos, según lo que mejor funciona a cada una. En este sentido, el Grupo C\&H (2016), hace referencia a la cultura de algunos países, y los tipos de liderazgo, que se desarrollan por lo general en cada uno, tales como:

El líder sincronizado: buscan consenso en la toma de decisiones y luego se encargan de convencer a los demás; tienden a ser prudentes $y$ se concentran más en posibles amenazas que en recompensas en regiones como China Continental, Corea del Sur, Japón, Tailandia, EEUU y una gran parte de Latinoamérica. Líder oportunista: Aquellos que piensan en objetivos por sí mismos y son flexibles respecto a cómo cumplirlos, son individualistas y se arriesgan a tomar decisiones ambiciosas, en países como Holanda, Alemania, Noruega, el Reino Unido o los países occidentales.

En este orden de ideas, el papel de los líderes debe estar acorde a las épocas y lugar donde viven, siendo flexibles para enfrentarse con éxito a los distintos entornos del mundo en constante cambio. Sobre esto Zamora, A. y Poriet, Y. (2012, 66), explican "La finalidad del liderazgo se relaciona con las actividades que promueven los líderes de estimular el pensamiento creativo y de estimular a los asociados para que se dediquen a trabajar en los objetivos de manera excelente". El líder, para poder lograr las metas debe saber cómo influir en la conducta de los seguidores, tomando en cuenta los objetivos planeados y las metas a alcanzar.

Dentro de este contexto, el liderazgo en las empresas venezolanas ha permitido impulsar a las organizaciones a niveles de eficiencia y eficacia organizacional, Loaiza y Pirela, $(2015,01)$ explican "Las organizaciones venezolanas están orientando parte de su estrategia a fortalecer el liderazgo emocional y resonante en sus directivos, gerentes y supervisores", el Liderazgo resonante, es un tipo de liderazgo que sintoniza con los sentimientos de las personas y los encauza en una dirección emocionalmente positiva. Este tipo de líderes sabe movilizar en los demás el estado de ánimo más adaptado a las circunstancias, utilizando su empatía para sintonizar con el tono emocional de sus subordinados. Cuando el líder no sabe empatizar ni interpretar adecuadamente las emociones ajenas genera disonancia $y$ trasmite innecesariamente mensajes perturbadores.

Dentro de estas empresas venezolanas, se encuentran las hoteleras. Una organización hotelera, tal como el resto de las empresas, poseen en su mayoría bases firmes en su metodología y administración, cuentan con gerentes que giran instrucciones precisas a los trabajadores para lograr con éxito las metas trazadas en la planificación, realizan toma de decisiones asertivas, mantienen altos niveles de productividad y rentabilidad, todo a través de un liderazgo cónsono con la realidad venezolana.

Contrario a lo expresado, si en una empresa hotelera se presentan dificultades y no existe una gerencia efectiva que presente un liderazgo firme a los empleados, los problemas existentes no se resolverán de manera definitiva, por el contrario siempre existirá un foco de complicaciones, que requerirá una nueva toma de decisiones. Sólo un gerente con un liderazgo efectivo que sepa conducir la empresa por una brecha exitosa, sorteará las irregularidades y deficiencias que en ella se presenten, tomando las decisiones que contribuyan al crecimiento de la organización y al buen desenvolvimiento de la misma en el mercado en el que se desarrolle.

En concordancia con lo planteado, en el Hotel Las Cabañas, ubicado en Los Guayos, Estado Carabobo se presenta una problemática relacionada directamente con la gerencia y la 
organización, especialmente con el tipo de liderazgo que viene afectando tanto el desempeño de los empleados como a la empresa en general. Esto debido al trato que el gerente tiene con el personal, en palabras de los empleados, quienes en conversaciones con los investigadores mencionaron han manifestado sentirse poco motivados, y no mantener comunicación asertiva con los gerentes; igualmente señalan la ausencia de falta de métodos de trabajo, planificación de actividades, toma de decisiones, así como consideran existe baja productividad.

En función a lo expuesto, a través de la presente investigación se analizará el liderazgo organizacional en el Hotel Las Cabañas, ubicado en el municipio Los Guayos, Estado Carabobo. Su repercusión al momento de adoptar técnicas de liderazgos no apropiadas las cuales pueden crear acciones negativas en el personal, afectando la producción y el prestigio del hotel.

En este sentido, dicha investigación, ofrece aportes a la empresa hotelera, que pueden servir de guía a las personas que ejercen función de líder, y estos sean aplicados al personal con el propósito de ser más productivos y efectivos en el ámbito laboral.

\section{MATERIALES Y METODO}

En relación al ámbito Metodológico, de acuerdo a Ramírez, $(2014,28)$ se refiere a "los métodos utilizados para hacer referencia al lugar del proyecto destinado para suministrar información sobre la manera cómo se va a realizar la investigación". En este sentido en cuanto al tipo de Investigación, fue una investigación cuantitativa ya que el estudio buscó la comprobación y la contrastación mediante la utilización del método científico.

El diseño que se utilizó fue de Campo. Este tipo de investigaciones, en palabras de Gómez $(2012,33)$ "Permite al investigador asegurarse de las condiciones fácticas, a través de su revisión y modificación, durante el despistaje puede sufrir modificaciones de espacio y tiempo y al reducirse a un sector puede originar una mayor precisión y seguridad. Sobre este aspecto se realizó la investigación en el Hotel Las Cabañas, Ubicado en Los Guayos, Estado Carabobo.

Así mismo, se abordó con un diseño descriptivo, en palabras de Bitte $(2012,10)$ estos son "Aquellos que están dirigidos a determinar cómo es, y como está la situación de las variables que se estudian en una población”. Es así como se realizó el análisis del liderazgo organizacional.

La población, estuvo conformada por 25 empleados del Hotel. En lo que se refiere a este tema Ramírez $(2014,45)$, indica "Una muestra es simplemente en general, una parte representativa de un conjunto, población o universo, cuyas características puede reproducir en pequeño lo más exactamente posible". Según el número que integra la población se considera Censal, de acuerdo a lo expresado por Hayes $(1999,28)$ "Es donde la Muestra es toda la población", en tal sentido se consideró toda la población como muestra es decir 25 empleados del Hotel las Cabañas.

Los métodos y técnicas de recolección de datos hacen referencia a la manera en la cual se recaudará la información, esto implica la manera como se establecerá la relación del investigador con el consultado". Para el estudio, se obtuvo la información directa de la fuente primaria; es decir, de los empleados del Hotel Las Cabañas. Se usó la entrevista oral estructurada, esta son la recopilación de los datos concretos, directos de un tópico de opinión estadístico, mediante el uso de cuestionario o entrevistas, con preguntas y respuestas precisas que permitieron hacer rápida la tabulación y análisis de la información.

En relación con la validación se utilizó el método de juicio de expertos sometiendo el instrumento a medición y revisión. La 
confiabilidad del instrumento se verificó mediante la prueba de Alpha de Crombach, una vez que el instrumento fue aprobado por los expertos para su aplicación, se realizó la prueba piloto, a un grupo del0 personas.

\section{RESULTADOS Y DISCUSIÓN}

En cuanto a la situación actual del liderazgo ejercido por la gerencia del Hotel Las Cabañas, municipio Los Guayos, estado Carabobo, sobre la motivación se apreció que solo el 5\% de los participantes se sienten motivados por el trabajo en sí. En lo referente a responsabilidad o desarrollo, supervisión, relaciones interpersonales, condiciones de trabajo y salario, un 67\% manifestó considerar estos elementos como pésimos; considerando necesario el diseño de herramientas administrativas para propiciar la cooperación voluntaria de los trabajadores o empleados, indispensables para el alto desempeño, generando confianza y propiciando nuevas ideas, los resultados obtenidos podrían incidir en el desempeño de los trabajadores dentro de la organización, lo que disminuirá o intervendrá en el logro de las metas organizacionales.

Al considerar los resultados sobre la toma de decisiones, se aprecia que el 100\% de los participantes la consideran como pésimas, por lo tanto las decisiones que se toman en esta organización, pueden estar interfiriendo en el desempeño del trabajador.

En lo que respecta a las características del liderazgo y comunicación los resultados permiten expresar que:

Comunicación lograda a través de encuestas, un $48 \%$ la considera regular y un $52 \%$ opina es pésima.

Comunicación canalizada a través de buzones, 20\% la cataloga de regular y un $80 \%$ opina es Pésima.
Transmisión de políticas, 100\% juzga es pésima. Instrucciones de trabajo, 100\% reseña es pésima.

Retroalimentación respecto a la corrección del desempeño, 68\% indicó es regular y 32\% aludió es pésima.

Observándose, existe debilidades pronunciadas en un aspecto de relevancia para el buen funcionamiento de la organización, como lo es la comunicación, esta permite la interacción humana, dentro del campo laboral, en donde es preciso que los mensajes recibidos o emitidos logren el cometido de trasmitir la información, y así se logren el desarrollo de las metas y objetivos de la organización, de tal forma que los resultados obtenidos vislumbran debilidades que deben ser atendidas con prontitud.

$\mathrm{Y}$ en lo referido a la toma de decisiones basadas en el problema o en la solución, el 100\% de los elementos muéstrales expusieron las consideraban pésimas. Evidentemente, la toma de decisiones en la vida de la organización general, se lleva a cabo partiendo de experiencias que no consideran riesgos significativos, siendo entonces necesario, aplicar los elementos centrales del proceso de decisión; ya que de acuerdo a los resultados, la toma de decisiones de los líderes de la organización no han sido lo más adecuados, para asegurar el funcionamiento de la empresa.

\section{CONCLUSIONES}

Partiendo del análisis de las teorías y conceptos sobre el Liderazgo Organizacional y los objetivos planteados en la presente investigación, se proyectan las siguientes conclusiones:

Sobre los factores internos y externos del liderazgo ejercido por la gerencia del Hotel Las Cabañas, se pudo construir una matriz FODA, permitiendo obtener una visión más clara de las 
fortalezas, oportunidades, debilidades y amenazas, que al tenerlas clara se posee una visión global e integral de la situación problemática. En cuanto a factores internos positivos o fortalezas tales como: la ubicación del Hotel, ya que es de fácil acceso para clientes de Venezuela y del extranjero que desconocen la geografía del estado, el conocimiento del mercado de hospedaje y esparcimiento, los recursos financieros para innovaciones, el mantenimiento y mejoramiento continuo de las áreas físicas, la calidad del producto final. En cuanto al apoyo logístico que requieran los clientes, se pudo evidenciar la experiencia del talento humano que se desempeña en la organización, las características especiales del producto que se oferta, la diversidad de productos y servicios, las cualidades del servicio que se considera de alto nivel. Lo que permite mantener número de clientes individuales y grupales y procesos técnicos y administrativos de calidad.

Al describir los factores externos positivos o las oportunidades, se encuentran las competencias débiles de la red hotelera del estado, mercado de esparcimiento mal atendido, necesidad de esparcimiento de las personas y clientes regulares, metas logradas por empresas de la zona que solicitan para celebración, servicios hoteleros y de esparcimiento, grupos de personas con necesidades de salud que ameritan uso de instalaciones tales como piscina y restaurante, convenciones y jornadas de empresas privadas que ameritan instalaciones del Hotel Las Cabañas y las visitas de clientes extranjeros al país y al estado Carabobo.

En lo atinente a los factores internos negativos o debilidades se encuentra: salarios bajos, falta de motivación de los recursos humanos, toma de decisiones realizada por el líder del hotel basadas en el problema o en la solución y la transmisión de políticas y objetivos de la organización realizada por el líder del hotel, las cuales en palabras de los trabajadores de la organización son de pésima calidad.

Sobre los factores externos negativos o las amenazas se pueden señalar: Cambios en el mercado actual a nivel nacional y estadal, variación y aumento reiterado de precio de insumos, necesarios para hospedaje y esparcimiento, débil poder adquisitivo de clientes nuevos y tradicionales, condiciones actuales del trasporte que conduce los clientes hacia el hotel, migración de clientes fuera del país y el retraso en entregas de empresas proveedoras del hotel.

Tomando en cuenta, que en los resultados presentados se evidencia que los mayores porcentaje los ocupa la alternativa Pésima, y sobre el tema Robbins, S. (2006,168), relata la Motivación es "la voluntad de ejercer altos niveles de esfuerzos hacia las metas organizacionales, condicionadas por la habilidad del esfuerzo de satisfacer alguna necesidad individual"; se concluye que los trabajadores de esta organización no se encuentra motivados a realizar las asignaciones que le corresponden, lo cual puede incidir en la ausencia de logro organizacional, por lo que es necesario realizar inversiones en innovaciones y actualizaciones sobre el liderazgo, como pilar fundamental en una organización, para favorecer y mantener la posición obtenida en el campo de la hotelería a nivel estadal, tambien, establecer sistemas de recompensas basado en la experiencia del talento humano que se desempeña en la organización invertida en el esparcimiento de las personas y clientes regulares.

Asi mismo, es importante promocionar las cualidades del servicio que se considera de alto nivel, que han mantenido el número de clientes individuales y grupales, dentro de potenciales 
clientes con débil poder adquisitivo, invertir parte de los recursos financieros destinados al mantenimiento, en solventar retraso en entregas de empresas proveedoras del hotel.

En cuanto al recurso humano, se hacce necesario incremento de salarios o bonificaciones, a través de horas extra para por visitas de clientes extranjeros, incentivar la motivación del recurso humano, mediante los beneficios que representan las visitas de clientes extranjeros y favorecer la motivación del recurso humano y el incremento del salario, a través de concursos o competencias de ideas para abordar clientes con débil poder adquisitivo.

Ante los planteamientos expuestos en esta investigación, se pueden sugerir las siguientes recomendaciones, que pueden contribuir a mejorar la imagen que proyectan en los trabajadores de una organización: inspeccionar las políticas empleadas actualmente por la organización, para el logro de los objetivos organizacionales, y comprobar las fortalezas y sortear las debilidades y amenazas presentes. Revisar periódicamente, misión, visión y objetivos de la organización, como estrategia que permita la concienciación, internalización de la importancia de la práctica de estos principios en donde el liderazgo ejercido en los trabajadores es fundamental.

Además, se sugiere crear mecanismos de participación para los trabajadores, de tal modo que los mismos puedan expresar y sentirse escuchados, mediante mecanismos como las reuniones de grupo y lluvias de ideas, y del mismo modo puedan aportar conocimientos al mejoramiento del trabajo. Por otra parte, es necesario difundir la importancia que le confiere la organización a los trabajadores, a través de medios de publicación organizacionales, tales como sistemas de carteleras, medios impresos, página web, entre otros. La finalidad es que exista la información suficiente y que se tome en consideración la relevancia que tiene para la organización la participación de cada trabajador.

En relación con, la motivación, se puede destinar una cartelera informativa para los trabajadores, donde se publique mensualmente, fechas de cumpleaños, aniversarios de los trabajadores, así como logros a nivel organizacional o personal. Además de ofrecer felicitaciones individualizadas por escrito y en forma verbal por cumple años y logros dentro de la organización.

Con respecto a la formación, se debe capacitar a los líderes instituidos en la organización, en liderazgo, sus formas, tipos, cualidades y estrategias, para fortalecer las capacidades de éstos y brindarles herramientas adecuadas de gestión para la responsabilidad que ostentan. A su vez, favorecer con cursos o capacitaciones a los supervisores, sobre el liderazgo, motivación y manejo del personal.

\section{REFERENCIAS}

Caraballo, N., Carrasco, Y. y Gil, I. (2013), Estudio del liderazgo basado en principios en una empresa del estado Carabobo. Presentado ante la Universidad de Carabobo para optar al grado de Licenciados en Relaciones Industriales.

Chiavenato, I. (2003) Administración de los Recursos Humanos. Editorial MacGrawHill.Book Company.D.F. México

Davis, K. y Newstrom, J. (2003).Comportamiento Humano en el trabajo. Décima Edición. Ed. Mc Graw Hill Interamericana. México

Daft, R. (2006) Introducción a la Administración. Cuarta Edición. Ediciones Thomson. México D.F

Diez, E. García, J. Martín, F y Periañez, R. (2004). Administración y Dirección. Editorial McGraw Hill

Grupo C\&H (2016) Los tipos de liderazgo en el mundo. Documento en línea disponible en http://grupocyh.com/los-tipos-de-liderazgoen-el-mundo 
Hayes, B. (1999) Diseños de encuestas, usos y métodos de análisis estadísticos. Editorial Oxford. México

Loaiza, C. y Pirela, L. (2015) Liderazgo en organizaciones venezolanas. Revista Venezolana de Gerencia (RVG) Año 20. № 69. Universidad del Zulia (LUZ)

López, Julio (2008). Motivación Laboral y Gestión de Recursos Humanos en la Teoría de Frederick Herzberg. Trabajo de tesis de maestría en la Facultad de Ciencias Administrativas de la Universidad Nacional Mayor de San Marcos. Lima Ecuador

Ochoa, Y. (2013) La cultura organizacional y el liderazgo como alternativas para lograr la eficiencia en los servicios prestados en la unidad de Atención Médica Integral de la UC, presentada para optar al grado de Maestría en Administración de empresas, mención Gerencia

Ramírez, T. (2014) Cómo hacer un Proyecto de Investigación. Reimpresión, Editorial Panapo de Venezuela. Caracas-Venezuela

Reyes, R. (2016) Estilo de liderazgo y su influencia en el comportamiento organizacional del personal obrero de la unidad educativa "José Gregorio Ponce Bello". Presentado en la Universidad de Carabobo para optar al título de Magíster en Administración de Empresas Mención Gerencia

Robbins, S. (2006). Comportamiento Organizacional. 10a . Edición. Pearson Educación. México

Robbins, S. y Coulter, M. (2005) Administración. Octava edición. Editorial Pearson Educación de México
Rodríguez (2013), Administración de recursos humanos. Conceptos de conducta interpersonal y caso. Ciencia y técnica. Tomo II. México: Grupo Noriega. Primera Re impresión

Sabino, C. (2009) El proceso de investigación. Ediciones Panapo, Caracas, Venezuela

Salazar, J.(2007) Liderazgos-Estilos. Universidad Gran Mariscal de Ayacucho. Cátedra: Liderazgo, Comunicación y Supervisión. Documento en línea disponible en COLACHO013.@hotmail.com

Valera, G. (2015) Análisis del nuevo modelo de liderazgo aplicado en una empresa del sector automotriz, como plataforma estratégica esencial en la alineación de los objetivos organizacionales. Presentado en la Universidad de Carabobo para optar al título de Especialista en gerencia de recursos humanos

Zamora, A. y Poriet, Y. (2012) Papel de los líderes y nuevas tendencias del liderazgo en el siglo XXI. Revista FACES · Volumen XVII · № 1 . Escuela de Relaciones Industriales Universidad de Carabobo. Documento en línea disponible en http://servicio.bc.uc.edu.ve/faces/revista/vol2 7nl.pdf

Zerpa, A. (2013), Liderazgo humanizador para una gerencia centrada en valores. Presentado en la Universidad de Carabobo para optar al título de Magíster en Gerencia Avanzada en Educación 